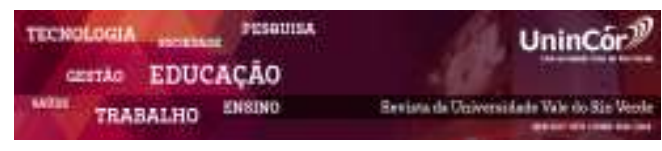

Revista da Universidade Vale do Rio Verde ISSN: 1517-0276 / EISSN: 2236-5362 Vol. 16 | n. 2 | Ano 2018

Luiz Henrique Mascarenhas Santos E.E.Vilmar Vieira Matos luizh.msdos@gmail.com

Maria do Socorro Mascarenhas Santos Universidade Estadual de Mato Grosso do Sul maria_mascarenhas@outlook.com

Leonilda Mascarenhas

Universidade Estadual de Mato Grosso do Sul leonilda@uems.br

Adriana Morais do Nascimento Matos Universidade Estadual de Mato Grosso do Sul adriana.nascimentomatos@gmail.com

Margareth Batistote Universidade Estadual de Mato Grosso do Sul margareth@uems.br

\section{OS RESÍDUOS SÓLIDOS URBANOS NO BRASIL E A POLÍTICA NACIONAL DE RESÍDUOS SÓLIDOS - LEI N ${ }^{\circ}$ \\ $12.305 / 2010$}

\section{RESUMO}

A produção dos resíduos sólidos urbanos (RSU) no Brasil é um problema que afeta diversos setores e, embora o perfil da sociedade venha se transformando ao longo do tempo, poucas alterações ocorreram na composição e na quantidade de resíduo gerado, levantando-se discussões acerca de temas tais como gestão e disposição correta, principalmente em relação ao cumprimento de políticas públicas. Com a implementação da Política Nacional de Resíduos Sólidos (PNRS) - lei $\mathrm{n}^{\mathrm{o}}$ $12.305 / 2010$ esperavam-se mudanças significativas para a disposição dos resíduos sólidos. Neste sentido, este estudo tem como objetivo discorrer sobre a gestão de resíduos sólidos no Brasil, sendo que as informações apresentadas e discutidas permitem uma visão atual sobre o desenvolvimento da gestão dos RSU no Brasil. São discutidos a expansão, a coleta e o tratamento dos RSU bem como a dificuldade da eliminação dos lixões e a mudança deles por aterros sanitários. Assim, embora tenha ocorrido melhorias no gerenciamento dos resíduos, ainda não estão de acordo com as alterações sugeridas pela PNRS.

Palavras-chave: Composição dos resíduos. Aterros sanitários. Sociedade. Lixo. Reciclagem.

\section{THE SOLID URBAN WASTE IN BRAZIL AND THE SOLID WASTE NATIONAL POLICY - LAW No. 12.305 / 2010}

\begin{abstract}
The production of urban solid waste (RSU) in Brazil is a problem that affects several sectors and, although the profile of society has been changing over time, few changes occurred in the composition and quantity of waste generated, rising discussions on issues such as its management and correct disposal, mainly in relation to compliance with public policies. With the implementation of the Solid Waste National Policy (PNRS) - law $\mathrm{n}^{\mathrm{o}} 12.305 / 2010$, significant changes were expected in the disposal of solid waste. In this sense, this study aims to discuss solid waste management in Brazil, and the information presented and discussed allows a current view on the development of PNRS management in Brazil. It discusses the expansion, collection and treatment of PNRS as well as the difficulty of disposal of the dumps and the change of them by landfills. Thus, although there have been improvements in waste management, they are still not in line with the changes suggested by the PNRS.
\end{abstract}

Keywords: Composition of residues. Landfills, Society. Garbage. Recycling. 


\section{INTRODUÇÂO}

A gestão apropriada dos Resíduos Sólidos Urbanos (RSU) é um desafio para todos os países, tanto desenvolvidos como em processo de desenvolvimento (Henry et al., 2006; Saikia e Nath, 2015). No Brasil a gestão dos resíduos cabe à sociedade e ao município (Brasil, 2010), devendo integrar o plano de saneamento e tendo como base legal as políticas públicas brasileiras, tais como a Política Nacional de Saneamento Básico, Lei no 11.445/2007, e a Política Nacional de Resíduos Sólidos (PNRS), Lei $\mathrm{n}^{\circ}$ $12.305 / 2010$.

Estas leis implicam na elaboração por parte dos municípios de um Plano Municipal de Gerenciamento Integrado de Resíduos Sólidos (PMGIRS) que, por sua vez, possuem papel de implementação da PNRS, garantindo que alguns aspectos referentes às questões dos RSU sejam regularizadas dentro dos setores público e privado, englobando, assim, toda a população. Contudo, 20 anos após a aprovação da Política Nacional de Saneamento Básico não houve mudanças significativas em relação à gestão de resíduos no país. (OLIVEIRA e JUNIOR, 2016).

Ademais, fatores como o crescimento da sociedade, a ocupação desordenada de terras e o consumo excessivo de produtos e bens de consumo tem acarretado sérios problemas aos órgãos competentes responsáveis pelo manejo dos resíduos, principalmente àqueles gerados nos centros urbanos, o que gerou discussões a respeito das políticas referentes à gestão de
Resíduos, seus métodos de coleta, tratamento e disposição corretos. Outro problema enfocado diz respeito à conscientização da população para a redução da produção de resíduos, ou seja, maneiras de reduzir os resíduos na fonte geradora, o que significa pensar nos resíduos antes mesmo deles serem gerados, buscando-se formas de não gerá-los (OLIVEIRA e JUNIOR, 2016).

Segundo Pereira (2004), "umas das maneiras de reduzir a quantidade de lixo gerado é o combate ao desperdício. A autora afirma que combater o desperdício não é fácil, mas que a educação ambiental pode facilitar no processo de mudança dos hábitos de consumo da população".

Ainda, de acordo com Vieira (2006), “o lixo tem várias designações e classificações, além de possuir a característica de gerar impactos ambientais". Neste contexto, os resíduos sólidos podem ser rotulados conforme sua natureza física podendo ser seco ou molhado; de composição química, classificando-se em orgânico ou inorgânico (Brasil, 2010; EU, 2008; Paraná, 2012; US-EPA, 2009) e, segundo a sua origem, também pode ser classificado em industrial, de saúde, da construção civil, entre outros.

O Brasil segue o modelo de gerenciamento dos RSU de países desenvolvidos como a União Europeia (EU, 2008) e EUA (USEPA, 2009) que possuem como princípios uma hierarquia que delibera como princípios a não geração, a redução, a reutilização, a reciclagem 
com o subsequente tratamento dos resíduos sólidos e a disposição final dos rejeitos, sendo que estes ocorrem depois de esgotadas as perspectivas tanto de tratamento como de recuperação dos resíduos por processos tecnológicos disponíveis e economicamente viáveis, restando com isso somente a disposição final ambientalmente adequada.

Diante deste contexto, este estudo possui como objetivo discorrer sobre a gestão de resíduos sólidos no Brasil.

\section{MATERIAL E MÉTODOS}

A pesquisa efetuada foi de natureza exploratória uma vez que se buscam informações sobre um assunto, e descritiva, já que investiga um determinado fenômeno ou problema coletando-se dados sobre as características e elementos intrínsecos a ele como parte do processo de conhecimento. Este estudo também apresenta uma interpretação qualitativa dos dados obtidos por meio da leitura de artigos, teses e dissertações relacionadas ao problema visando-se promover um melhor entendimento sobre o tema.

\section{RESULTADOS E DISCUSSÃO}

De acordo com dados da ABRELPE (2014) e IBGE (2013), no Brasil as regiões urbanas mais populosas eram o Nordeste e o Sudeste com 28 e $42 \%$ de habitantes, conforme figura 1.

As projeções demográficas para o período de 2000 a 2060 indicam que a população brasileira atingirá seu máximo em 2042, com aproximadamente 228,4 milhões de habitantes, fato que reflete na coleta dos RSU. O aumento da população também está diretamente relacionado a questões econômicas, isso porque existe um custo para que este procedimento seja realizado sistematicamente. Além disso, a coleta seletiva não deve ser apenas voltada para os resíduos recicláveis como ocorre atualmente, devendo ser fruto também da conscientização da sociedade para a matéria orgânica (IBGE, 2013).

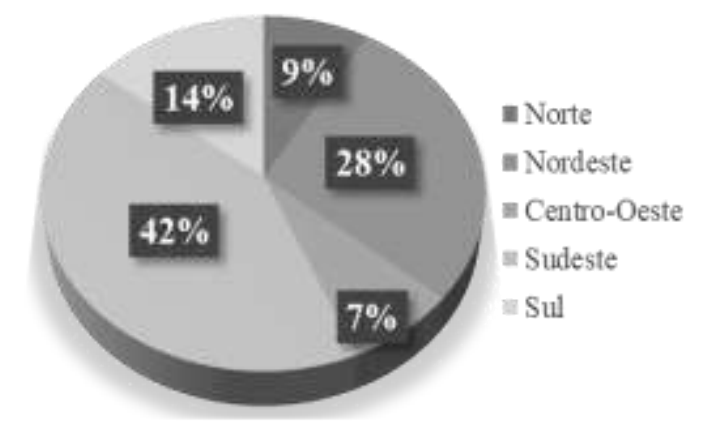

Figura 1. Estimativa da população no ano de 2013.Fonte: ABRELPE (2014) e IBGE (2013).

A figura 2 apresenta o quantitativo aproximado de resíduos gerados nas regiões do Brasil, sendo proporcionais ao número de habitantes, ou seja, as regiões mais populosas geram maior quantidade de resíduos.

É notório que as regiões Nordeste e Sudeste juntas geram um montante de $75 \%$, necessitando-se tanto de uma infraestrutura que suporte a consequente geração de resíduos bem como é necessária uma gestão estratégica que propicie uma coleta e uma destinação adequadas para estes resíduos. Contudo, existem fatores que interferem no cenário que seria ideal, haja vista que uma parcela dos RSU produzidos não é coletada e que a geração dos resíduos possui uma ligação direta com o poder aquisitivo da população. 
As pessoas com maior poder aquisitivo tendem a produzir uma quantidade elevada de resíduos. Deste modo, pode-se considerar a geração de resíduos sólidos como um indicativo social e econômico. Segundo Ojeda-Benítez et al. (2008) e Suthar e Singh (2015), a geração de resíduos está relacionada com fatores como os hábitos de consumo, padrão de vida, a cultura e a renda familiar.

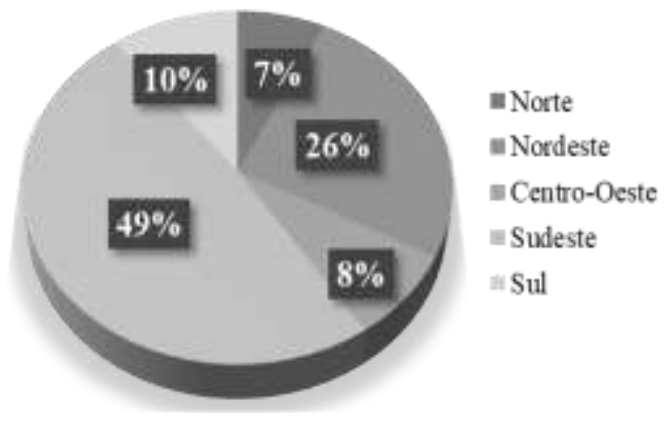

Figura 2. Quantidade de RSU gerado em toneladas/ dia no ano de 2013.Fonte:ABRELPE (2014) e IBGE (2013).

Quantificar os RSU gerados não é uma tarefa fácil, pois existem vários entraves que delimitam as pesquisas sobre estes índices, e vários problemas foram observados no decorrer deste estudo. Observou-se que a quantificação na geração não oferece números reais uma vez que os resíduos sólidos podem ser dispostos de forma irregular, ou pode ocorrer a coleta informal e, ainda, os sistemas de coletas públicas muitas vezes mostram-se insuficientes, acarretando em uma coleta parcial dos resíduos sólidos (IPEA, 2012).

Em comparação, nos países da Europa e dos Estados Unidos ocorre uma maior porcentagem de reciclagem dos RSU, já em países em desenvolvimento os resíduos são formados, em uma grande parcela, por matéria orgânica (Campos, 2012). No Brasil, entretanto, este perfil vem se alterando gradativamente por conta da mudança de hábitos da população, sendo que nos últimos anos vem ocorrendo um aumento na geração de recicláveis (Brasil, 2012).

De acordo com a Tabela 1, a geração de resíduos por habitante demonstra que em regiões mais desenvolvidas ocorre uma maior produção de resíduos. Assim, as regiões com maior taxa de geração são Sudeste e Centro- Oeste, com 1.21 e $1.1 \mathrm{~kg} / \mathrm{hab} / \mathrm{dia}$ contrastando com a região Sul, que apresentou $0.76 \mathrm{~kg} / \mathrm{hab} / \mathrm{dia}$ na geração de resíduos sólidos urbanos, isso porque esta região possui uma gestão integrada e estratégica sólida que contempla as necessidades das cidades.

Tabela 1. Quantidade de RSU gerado em

\begin{tabular}{lc}
\hline Regiões & $\begin{array}{c}\text { Índice } \\
(\mathbf{K g} / \mathbf{h a b} / \mathbf{d i a})\end{array}$ \\
\hline Norte & 0.89 \\
Nordeste & 0.96 \\
Centro-Oeste & 1.11 \\
Sudeste & 1.21 \\
Sul & 0.76 \\
\hline g/habitantes/dia no ano de 2013.
\end{tabular}

Fonte: ABRELPE (2014) e IBGE (2013).

Em relação à coleta dos resíduos em áreas urbanas, conforme demonstra a figura 3, alcança-se um grande percentual de residências nas regiões brasileiras, sendo que as regiões Sudeste, Centro-Oeste e Sul estão entre as que realizam de forma mais abrangente a coleta de resíduos apresentando índices de 22 e $21 \%$.

De acordo com a ABRELPE (2014), os dados comparativos entre a quantidade gerada e a quantidade coletada mostram que deixam de ser coletados aproximadamente 20 mil toneladas por dia e que provavelmente estes resíduos tiveram um destino inapropriado. 


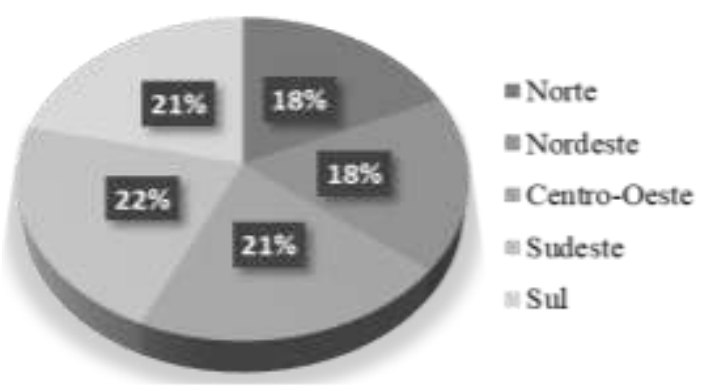

Figura 3. Percentual de coleta de RSU gerados no ano de 2013. Fonte: ABRELPE (2014) e IBGE (2013).

Mas, embora sejam constatados diversos problemas e precarização no descarte correto, por outro lado a destinação final dos resíduos ao longo dos anos vem se tornando mais efetiva, de acordo com a análise cronológica da Figura 4, na qual é possível visualizar o panorama de comparação no decorrer dos anos das disposições dos resíduos nos locais de destino.

Em 1989 a maior parte dos RSU iam para os lixões, o que corresponde a cerca de $88 \%, 10 \%$ eram descartados em aterros controlados e $1 \%$ para os aterros sanitários. Já em 2008 observa-se que os RSU apresentavam uma destinação ambientalmente correta, ainda que se deparasse com uma parcela relativamente alta de resíduos que vão para os lixões, algo em torno de $55 \%$.

De acordo com Jacobi e Besen (2011), houve um aumento das disposições dos RSU em aterros sanitários entre 2000 e 2008 e, isso se deve ao fato de que as 13 cidades mais populosas do Brasil, têm a maior parcela de seus resíduos dispostos em aterros sanitários. Alguns dos fatores que contribuíram para dados mais positivos em relação aos RSU foi a evolução nos conceitos e na eficiência da legislação em relação à destinação correta dos resíduos bem como dos tratamentos que podem mitigar a disposição inadequada. Nesse sentido, o tratamento e a gestão de resíduos sólidos urbanos devem ser observados com cautela e planejamento, principalmente devido ao impacto sobre o meio ambiente, salienta SantibañezAguilar et al.(2013).

Os processos mais difundidos para os resíduos são a reciclagem e a compostagem para resíduos orgânicos (Brasil, 2010).

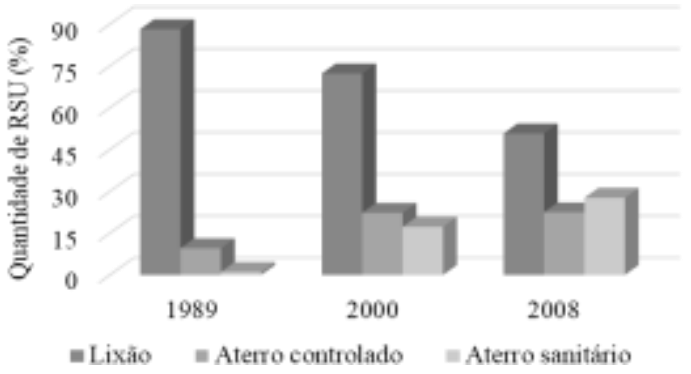

Figura 4. Destino final dos RSU entre os anos de 1989 a 2008. Fonte: IBGE (2013).

Quanto à disposição final dos RSU, que foi identificada como adequada e inadequada, a Figura 5 apresenta a comparação entre os anos de 2012 e 2013. Nota-se que houve pouca diferença de um ano para outro, contudo, ressalta-se que a taxa de descarte dos resíduos de modo inadequado foi de aproximadamente $45 \%$, o que sugere a sua deposição em locais sem controle, o que pode acarretar impactos ambientais.

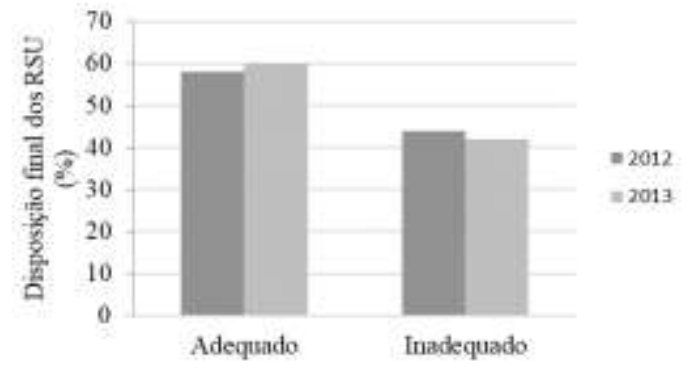

Figura 5. Disposição dos RSU. Fonte: ABRELPE (2014). 
Esta deposição dos RSU pode ter uma relação direta com a sua composição. A média da composição dos RSU do Brasil é formada por $32 \%$ de material reciclável, $51 \%$ de matéria orgânica e $17 \%$ de outros materiais que são tidos como rejeitos que não são recicláveis ou se mostram inviáveis para a reciclagem (Figura 6). E, segundo Andrade e Ferreira (2011), independente do tratamento ou técnica a ser utilizada, é necessário realizar a caracterização da composição gravimétrica dos resíduos.

Um dos poucos estudos sobre aspectos econômicos da reciclagem foi realizado pelo IPEA em 2010, indicando que o país perde, anualmente, cerca de $\mathrm{R} \$ 8$ bilhões ao enterrar o lixo que poderia ser reciclado.

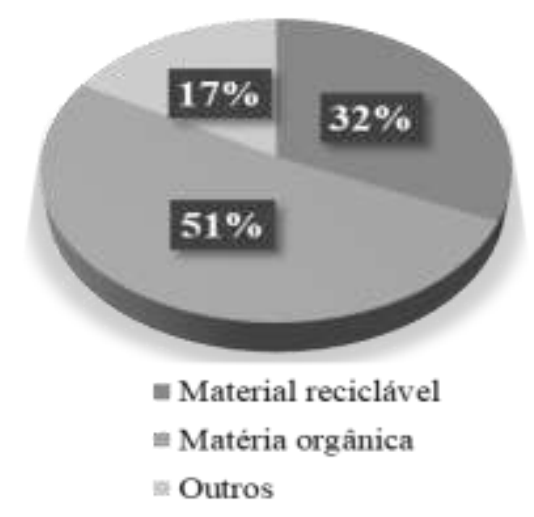

Figura 6. Composição dos RSU. Fonte:(Brasil, 2012).

\section{CONSIDERAÇÕES FINAIS}

Em relação à geração dos RSU no Brasil, com o aumento da população a tendência é de aumentar também a geração de resíduos, contrariando-se os conceitos da não geração, da redução e da reutilização.

Ainda que a reciclagem e a compostagem venham aumentando no Brasil a coleta seletiva precisa ser aperfeiçoada e estudada para que se torne mais eficiente, melhorando a sua abrangência e diminuindo os custos, incentivando, principalmente a participação dos catadores, os quais realizam o trabalho de seleção de materiais aproveitáveis, bem com incentivar a sociedade neste processo de separar os resíduos orgânicos e recicláveis nas suas residências.

Em contrapartida, a disposição final dos RSU de lixões e aterros controlados, formas inadequadas, deveria ter sido encerrada até o ano de 2014, sendo dispostos a partir de então em aterros sanitários, porém isto não ocorreu na maioria dos municípios brasileiros.

Assim, com a Proposta do Plano Nacional de Resíduos Sólidos e com os novos planos municipais de gerenciamento integrado de resíduos sólidos, a expectativa é que ocorra o crescimento do mercado dos recicláveis no Brasil, baseado na coleta seletiva e na formação de cooperativas com investimentos públicos e empresariais, principalmente no que diz respeito à logística reversa e outras ferramentas relacionadas à implementação da responsabilidade compartilhada pelo ciclo de vida dos produtos (Brasil, 2012).

\section{REFERÊNCIAS}

ASSOCIAÇÃO BRASILEIRA DE EMPRESAS DE LIMPEZA PÚBLICA E RESÍDUOS ESPECIAIS ABRELPE. Panorama dos Resíduos Sólidos no Brasil - 2013. 2014. Disponível em: http://www.abrelpe.org.br/Panorama/panorama2013.p df. Acesso em: jan. 2018.

ANDRADE, R. M.; FERREIRA, J. A. A Gestão de resíduos sólidos urbanos no Brasil frente às questões da globalização. Revista Eletrônica do Prodema REDE, v. 6, n. 1, p. 7-22, 2011. 
BRASIL. Lei no 12.305, de 2 de agosto de 2010. Institui a Política Nacional de Resíduos Sólidos; altera a Lei no 9.605, de 12 de fevereiro de 1998 e dá outras providências. Diário Oficial [da] União, Brasília, 03 ago. 2010.

Ministério das Cidades. Secretaria Nacional de Saneamento Ambiental. Lançamento Diagnóstico Resíduos sólidos 2013. 2013. Disponível em: http://www.snis.gov.br/index.php/component/content/ article?id=108. Acesso em: jan. 2018.

Ministério do Meio Ambiente; ICLEI

BRASIL. Planos de gestão de resíduos sólidos: manual de orientação - apoiando a implementação da política nacional de resíduos sólidos - do nacional ao local. 2012. Disponível em: http://www.mma. gov.br/estruturas/182/_arquivos/manual_de_residuos_ solidos3003_18 2.pdf. Acesso em: jan. 2018.

Proposta do plano nacional de resíduos sólidos. Brasília: Ministério do Meio Ambiente. Disponível em: http://www.mma.gov.br/port /conama/ reuniao/dir1529/PNRS_consultaspublicas.pdf. 2012. Acesso em: jan, 2018.

CAMPOS, H. Renda e evolução da geração per capita de resíduos sólidos no Brasil. Engenharia Sanitária e Ambiental, v. 17, n. 2, p. 171 -180, 2012.

HENRY, R. K.; YONGSHENG, Z.; JUN, D. Municipal solid waste management challenges in developing countries--Kenyan case study. Waste management, v. 26, n. 1, p. 92-100, 2006.

INSTITUTO BRASILEIRO DE GEOGRAFIA E ESTATÍSTICA - IBGE. Perfil dos municípios brasileiros 2013. 2014. Disponível em: http://cidades.ibge.gov.br/xtras/home.php. Acesso em: jan. 2018.

INSTITUTO BRASILEIRO DE GEOGRAFIA E ESTATÍSTICA - IBGE. Projeção da população do Brasil por sexo e idade para o período de 2000 / 2060. 2013. Disponível em: http://www.ibge.gov.br /home/estatistica/populacao/projecao_da_populacao/2 013/. Acesso em: jan. 2018.

\section{INSTITUTO DE PESQUISA ECONÔMICA} APLICADA - IPEA. Diagnóstico dos resíduos sólidos urbanos - relatório de pesquisa. 2012. Disponível em: http://www.ipea.gov.br/agencia /images/stories/PDFs/relatoriopesquisa/_relatorio_resi duos_solidos_urbanos.pdf. Acesso em: jan. 2018.

JACOBI, P. R.; BESEN, G. R. Gestão de resíduos sólidos em São Paulo: desafios da sustentabilidade. Estudos Avançados, v. 25, n. 71, p. 135-158, 2011.

KARAK, T.; BHAGAT, R. M.; BHATTACHARYYA, P. Municipal solid waste generation, composition, and management: The world scenario. Critical Reviews in Environmental Science and Technology, v. 42, n. 15, p. 1509-1630, ago. 2012.

OLIVEIRA, T. B.; JUNIOR, A. C. G. O planejamento municipal na gestão dos resíduos sólidos urbanos e na organização da coleta seletiva. Engenharia Sanitária e Ambiental, v. 21, n. 1, 2016.

OJEDA-BENÍTEZ, S.; VEGA, C. A. DE; MARQUEZ-MONTENEGRO, M. Y. Household solid waste characterization by family socioeconomic profile as unit of analysis. Resources, Conservation and Recycling, v. 52, n. 7, p. 992-999, 2008.

PARANÁ. Unidades de triagem e compostagem de resíduos sólidos urbanos. 2012. Disponível em: http://www.mpgo.mp.br/portal/arquivos/2013/06/27_a postila_rsu_mppr.pdf. Acesso em: jan. de 2018.

SAIKIA, D.; NATH, M. J. Integrated solid waste management model for developing country with special reference to Tezpur municipal area, India. International Journal of Innovative Research \& Development, v. 4, n. 2, p. 241-249, 2015.

SANTIBAÑEZ-AGUILAR, J. E. et al. Optimal planning for the sustainable utilization of municipal solid waste. Waste management, v. 33, n. 12, p. 2607-22, 2013

SUTHAR, S.; SINGH, P. Household solid waste generation and composition in different family size and socio-economic groups: a case study. Sustainable Cities and Society, v. 14, p. 56-63, 2015

UNITED STATES. Environmental Protect Agency. US-EPA. Hazardous waste characteristics: a userfriendly reference document. 2009. Disponível em: http://www.epa.gov/osw/hazard/wastetypes/wasteid/ch ar/hw-char.pdf. Acesso em: jan. 2018.

\section{Luiz Henrique Mascarenhas Santos}

Bolsista PIBIC-Jr /CNPQ.

\section{Leonilda Mascarenhas}

Graduanda em Direito - UEMS; Especialista em Estudos Linguísticos - UEMS; Bacharel em Letras UFMS.
Maria do Socorro Mascarenhas Santos

Mestre em Recursos Naturais - UEMS; Especialista em Biotecnologia - UCDB; Tecnóloga em Produção Sucroalcooleira - UEMS; Tecnóloga em Gestão Ambiental - UCDB. 


\section{Adriana Morais do Nascimento Matos}

Mestre em Recursos Naturais - UEMS; Engenheira

Agronoma - Anhanguera; Biologa - UNIGRAN;

Graduação em Manejo e Nutrição Animal UNIGRAN.

\section{Margareth Batistote}

Pós-doutorado e Doutorado em Biotecnologia - UEP

"Júlio de Mesquita Filho"; Mestre em Microbiologia - UEL; Biologa - UCDB. 